\title{
Editorial
}

\section{International Conference on Structural Engineering Dynamics 2013}

\author{
Nuno M. M. Maia and Miguel M. Neves \\ LAETA, IDMEC, Instituto Superior Técnico, Universidade de Lisboa, Lisboa, Portugal \\ Correspondence should be addressed to Nuno M. M. Maia; nmaia@dem.ist.utl.pt
}

Received 12 May 2014; Accepted 12 May 2014; Published 10 September 2014

Copyright (C) 2014 N. M. M. Maia and M. M. Neves. This is an open access article distributed under the Creative Commons Attribution License, which permits unrestricted use, distribution, and reproduction in any medium, provided the original work is properly cited.

The last edition of the International Conference on Structural Engineering Dynamics (ICEDyn) 2013 took place in Sesimbra, a tiny fishing village in the Blue Coast Portugal, on the 17th-19th of June 2013, and was organized by the Instituto Superior Técnico (IST), from the University of Lisbon, and the Instituto de Engenharia Mecânica (IDMEC).

As it happened in previous editions of this biennial scientific event, the conference embraces a large spectrum of subjects that attract the interest of a wide community devoted to engineering dynamics, where theory and experiment mix very well. Common to all ICEDyn conferences is the high scientific standard of the submitted articles. Senior scientists, representatives of the industrial community, and young students doing their Master or Ph.D. studies were encouraged to meet and develop a healthy and stimulating interaction around the same kind of interests and objectives.

Four international renowned scientists addressed keynote lectures that preceded and framed the parallel sessions that followed them: "Operational modal analysis" (R. Brincker, Aarhus University, Denmark); "On the renewed interest for the wave propagation approach to structural dynamics" (J. R. F. Arruda, Universidade Estadual de Campinas, Brazil); "Spatial information in autonomous modal parameter estimation" (R. J. Allemang, University of Cincinnati, USA); and "Regular and chaotic dynamics of flexible plates" (J. Awrejcewicz, Łódź University of Technology, Poland). The parallel sessions covered damage/SHM (3 sessions), active control (2 sessions), experimental/operational modal analysis (2 sessions), sound and vibration generated by moving loads ( 2 sessions), aeronautics and aerospace
(2 sessions), smart materials in structural dynamics (2 sessions), modelling, civil structures, nonlinear dynamics, analytical methods, connecting analysis and tests, and damping and updating ( 2 sessions).

This special issue has been carefully prepared, in order to meet the high standards of this publication and to reflect the excellent outcome of ICEDyn 2013. Those authors who had manifested an interest in publishing in SAV submitted improved versions of their articles, which have passed through a rigorous process of peer-reviewing (with at least two reviewers and most of them with three reviewers); this has contributed to the enrichment of the scientific quality of those articles. From the initial 43 papers, a total of 29 papers have been finally selected to integrate the present issue, covering various areas. It was a pleasure and an honor for us to serve as Guest Editors for this special Issue. We hope that readers will find the special issue exciting as well as useful for stimulating further research activities and conjecturing future trends in this area of structural dynamics.

\section{Acknowledgments}

Acknowledgments are due to all contributing authors and coauthors, to the reviewers for all their effort, time, and collaboration, and to our colleague of the Organizing Committee Professor Chedas Sampaio, for their support of this 2013 special issue.

Nuno M. M. Maia Miguel M. Neves 

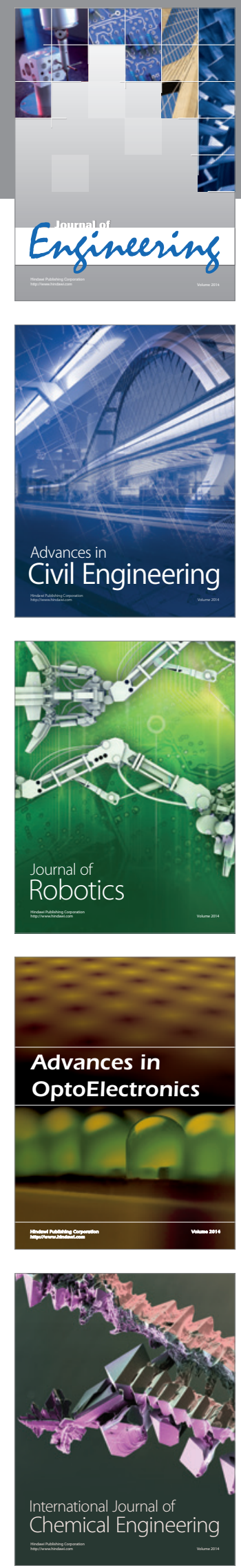

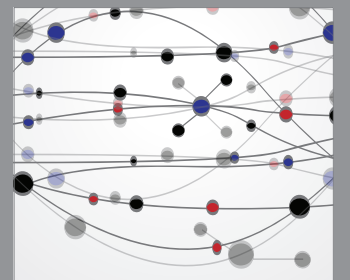

The Scientific World Journal
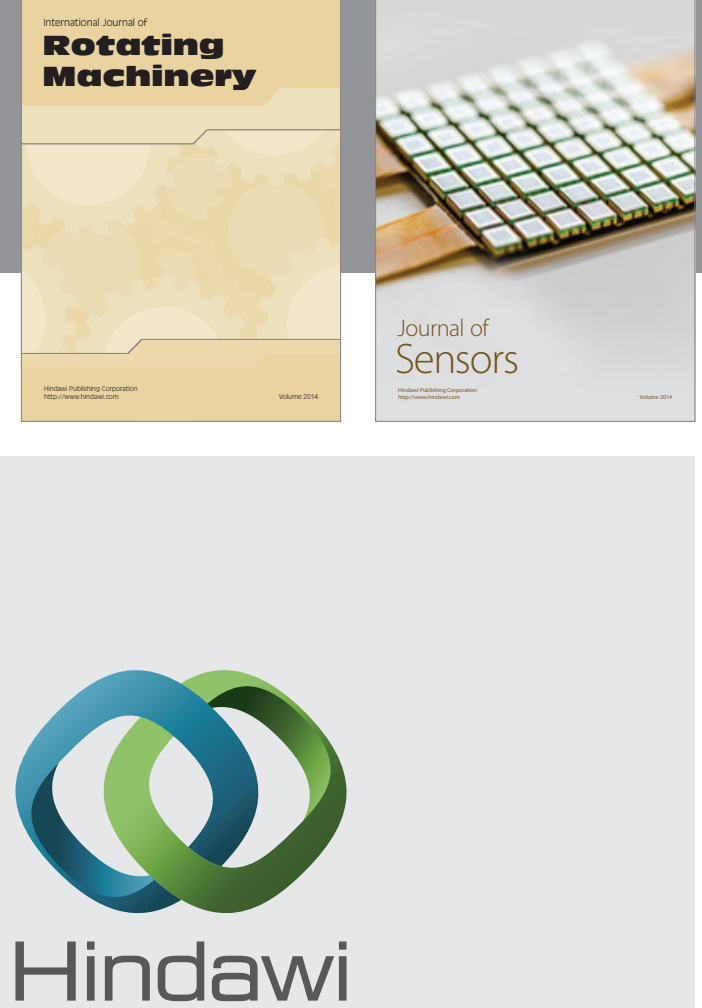

Submit your manuscripts at http://www.hindawi.com
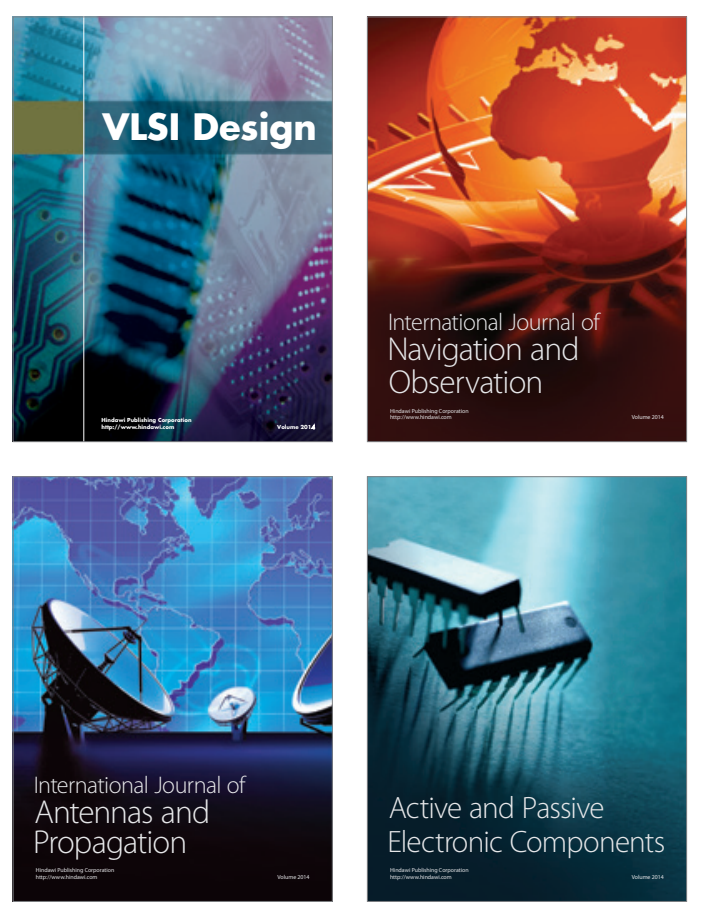
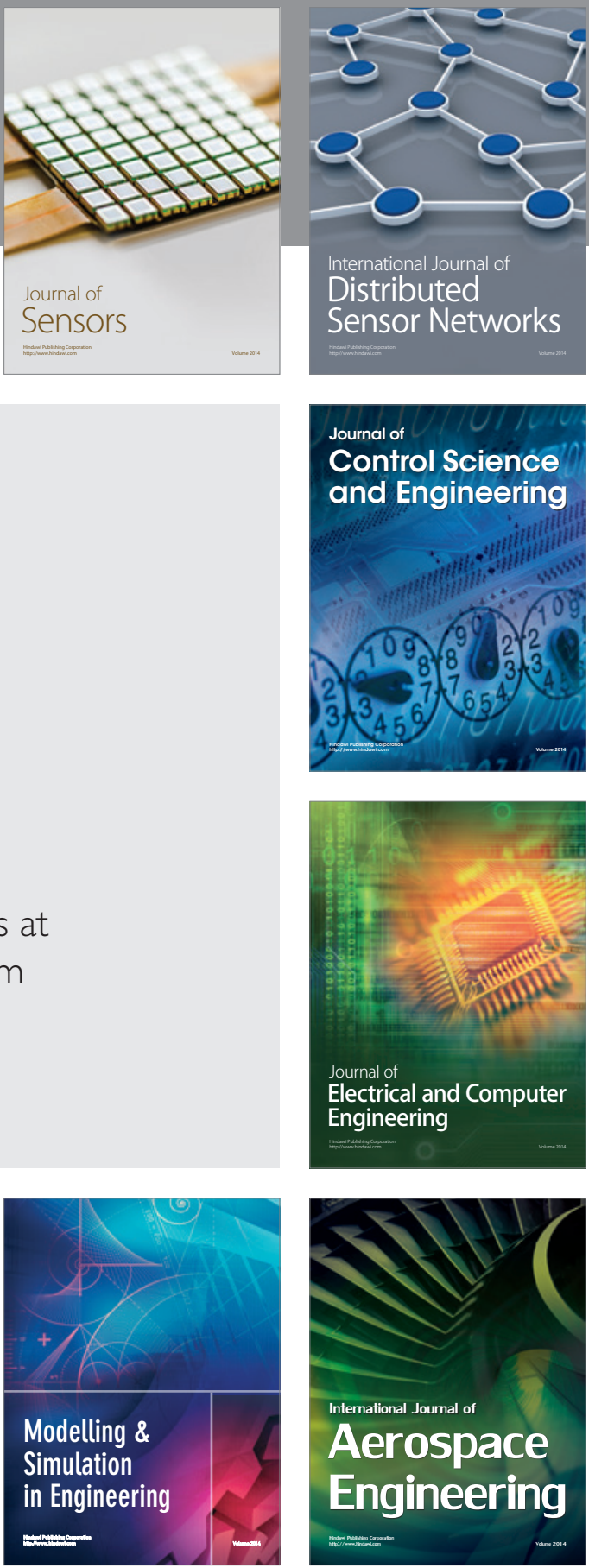

Journal of

Control Science

and Engineering
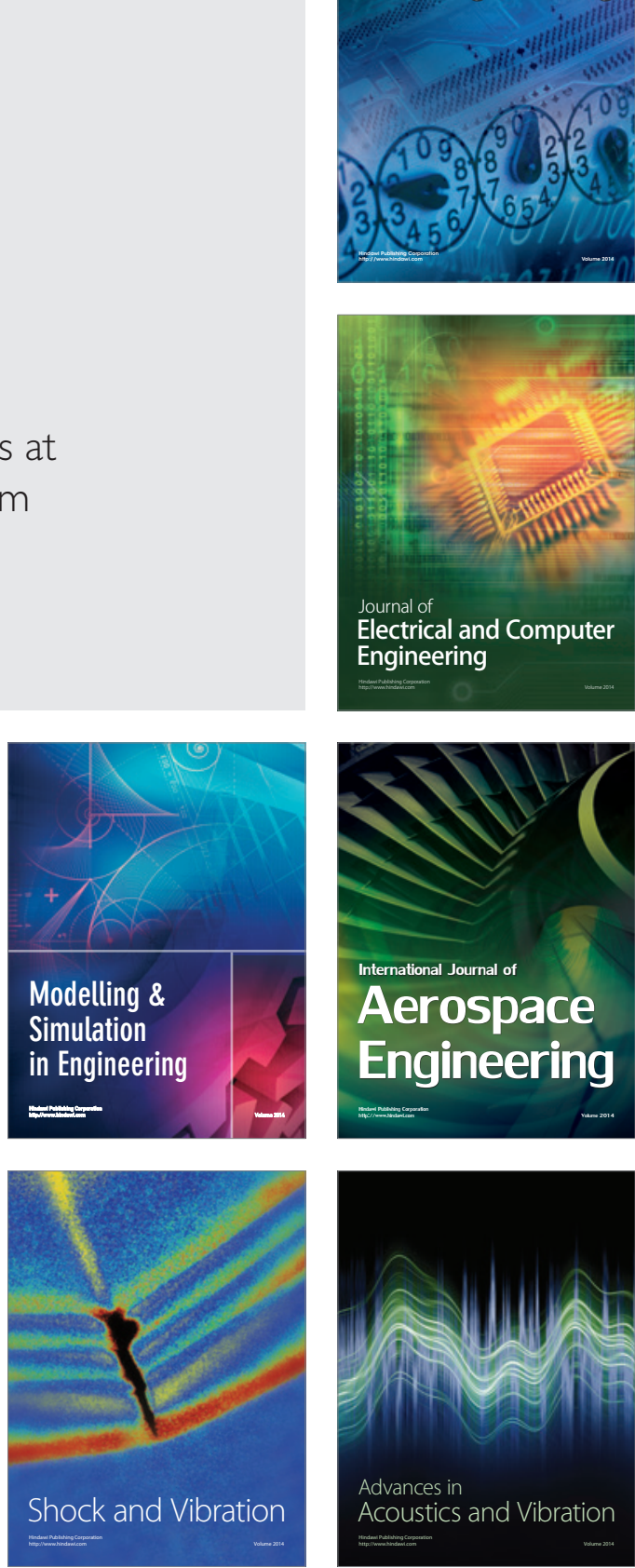